\title{
COMPETENCIA SOCIOEMOCIONAL EN EL AULA: CARACTERÍSTICAS DEL PROFESOR QUE FAVORECEN LA MOTIVACIÓN POR EL APRENDIZAJE EN ALUMNOS DE ENSEÑANZA SECUNDARIA OBLIGATORIA
}

\author{
SOCIAL AND EMOTIONAL COMPETENCIES IN THE CLASSROOM: \\ TEACHER CHARACTERISTICS THAT FOSTER MOTIVATION \\ FOR LEARNING IN ESO STUDENTS
}

\author{
$M^{a}$ Cristina Núñez del Río* y Mónica Fontana Abad** \\ Universidad Complutense de Madrid
}

\begin{abstract}
RESUMEN
No se puede negar el incremento en los diez últimos años de investigaciones y publicaciones centradas en la Competencia Socioemocional. En concreto, este estudio aborda uno de los procesos que, según Goleman (1996), forma parte del constructo Inteligencia Emocional: la motivación. Numerosos estudios tratan su relación con el rendimiento y el fracaso escolar (González, Mendiri y Arias, 2002; Brier, 2006). El aumento de los índices de desmotivación en las aulas, unido a un rendimiento académico cada vez menor en algunos grupos de alumnos - a los que se unen aspectos y situaciones familiares disfuncionales - justifica este estudio acerca de uno de los factores que más incidencia puede tener en los alumnos y sobre el que se puede intervenir: se trata de las características de los profesores que son percibidas como motivadoras por sus alumnos. En concreto, el estudio aborda el análisis de las diferencias en función de los diferentes cursos, el sexo y los factores de motivación según la Escala de Motivación Académica (EMA, Manassero y Vázquez, 1997, 1998). Para ello, se presentan los primeros hallazgos de un estudio, con una muestra incidental de 350 alumnos de ESO, pertenecientes a dos colegios concertados de diferentes áreas de Madrid. El trabajo concluye con algunas pautas de intervención para los profesores, que se consideran recomendables en la actuación en las aulas.
\end{abstract}

\footnotetext{
* Doctora en Educación por la Universidad Complutense de Madrid y Profesora Titular Interina del Departamento de MIDE, de la UCM, con líneas de investigación en el ámbito del Diagnóstico, Orientación Educativa y Atención a la Diversidad. E-mail: mcnunez@edu.ucm.es.

** Doctora en Educación por la Universidad Complutense de Madrid y Profesora Titular Interina del Departamento de MIDE, de la UCM, con líneas de investigación en el ámbito de la Orientación Educativa y Familiar y Calidad de la educación. E-mail: mfontana@pdi.ucm.es.
} 
Palabras clave: competencia socioemocional; motivación, rendimiento académico, Educación Secundaria Obligatoria, profesorado.

\begin{abstract}
It can't be denied that, in the last ten years, research and publications focusing on Social and Emotional Competencies is increasing. This study addresses one of the processes, which according to Goleman (1996), is part of the Emotional Intelligence construct: motivation. Numerous studies deal with its relationship with performance and school failure (Gonzalez, Mendiri and Arias, 2002; Brier, 2006). The increased rates of demotivation in the classroom, coupled with an increasingly lower academic performance in some groups of pupils, together with dysfunctional family situations, justify this study about one of the factors with more impact on students: the characteristics of teachers who are perceived as motivating by the students. Specifically, in this paper, the differences taking into account the courses, the sex and the motivational factors according to the Academic Motivation Scale (EMA, Manassero and Vazquez, 1997, 1998) will be analyze. For this propose, the first findings of a study, with an incidental sample of 350 students from two schools in different areas of Madrid, will be presented. The paper concludes with some recommended guidelines for intervention for teachers in the classroom.
\end{abstract}

Key words: social and emotional competencies; motivation; academic performance; secondary education teacher.

\title{
Introducción
}

El aumento de los índices de fracaso escolar en el aula y la innegable disminución del rendimiento académico han llevado a numerosos investigadores a interesarse por los factores que influyen en la motivación de los alumnos por el aprendizaje (Broc, 2006; Schiltz, 2004; González, Mendiri y Arias, 2002; Manassero y Vázquez, 1997, 1998; Wentzel, 1998; Menec, 1997).

La motivación es un constructo no observable directamente y cuya importancia reside, entre otros factores, en su capacidad explicativa y predictiva de la conducta humana en diferentes situaciones y contextos (Manassero y Vázquez, 1998). Comúnmente se entiende por motivación aquella fuerza que mueve al ser humano a realizar algo determinado, implicando de lleno la dimensión volitiva, el querer (Bueno, 2004; Menec, 1997).

Con los años, además de una perspectiva individual, se han ido adoptando modelos sistémicos que abordan los aspectos relacionales del constructo individual. De momento, la investigación sobre las conductas de los profesores que promueven activamente la motivación para aprender ha sido relativamente escasa.

En la literatura sobre los factores que influyen en la motivación por aprender surgen dos claras dimensiones: la social — que trata la relación con los profesores en el contexto escolar - y la emocional — centrada en variables afectivas, como el apoyo recibido y la vinculación con la escuela-.

Sekiro y Fantuzzo (2005), en un estudio con una muestra de 242 alumnos de secundaria, observaron cómo el área cognitiva mantenía una relación consistente con la habilidad matemática y la lectura temprana, mientras que la dimensión socioemocional correlacionaba con la persistencia, las interacciones prosociales y la motivación por aprender. 
Las relaciones sociales han sido incluidas como variable predictora de gran variedad de resultados escolares. La misma relación profesor-alumno se ha señalado como uno de los factores más favorecedores de las competencias socioemocionales y de la motivación por aprender, al constituirse en uno de los principales puntos de apoyo para los alumnos (Patrick, Mantzicopoulos, Samarapungavan y French, 2008; Wentzel, 1998; Resnick, Bearman, Blum, Bauman, Harris, Jones, Tabor, Beuhring, Sieving, Shew, Ireland, Bearinger, y Udry, 1997). Pobres relaciones con los profesores y pobres vínculos con la escuela conllevan una menor adaptación socioemocional (Murray y Greenberg, 2000). Wooley, Kol y Bowen (2009) han demostrado cómo la misma relación profesor-alumno, y su calidad, afecta al rendimiento de los estudiantes, examinando los vínculos directos e indirectos de estas relaciones. En concreto, el apoyo que brinda el profesor, a nivel escolar y emocional, se asocia con la conducta y el nivel de satisfacción con la escuela de los alumnos e, indirectamente, con el tiempo empleado y la calidad en la realización de las tareas escolares. En el estudio de Wentzel (1997) el apoyo de los profesores, percibido por los alumnos, también predijo resultados respecto de la motivación. Se señalaron como aspectos más relevantes: Los estilos de interacción democráticos, el desarrollo de expectativas positivas hacia las conductas de los alumnos a partir de sus diferencias individuales, la práctica de una actitud cuidadosa hacia el trabajo de cada alumno y la facilitación de feedback constructivo a los alumnos.

Puede afirmarse, por tanto, que el estudio de las relaciones profesor-alumno y las experiencias de vinculación positivas hacia la escuela están emergiendo como una importante área de estudio.

Un gran número de investigaciones que examinan estas relaciones parten de tres bases teóricas explicativas: La Teoría del Apego (Bowlby, 1982), la Teoría del Control Social (Hirsch, 1969) y la Teoría del Desarrollo de Sistemas (Ford y Lerner, 1992). Comparten el objetivo común demostrar cómo las experiencias relacionales y sociales influyen en el desarrollo humano: los niños no se desarrollan solos y, por ello, las relaciones sociales y las experiencias que tienen en diferentes contextos — siendo prioritarios la escuela y el aula - influyen en la adaptación conductual, emocional y motivacional (Murray y Greenberg, 2000; Pianta, 1999; Resnick y cols. 1997). Considerando la metodología de estudio, las investigaciones abordan la motivación o las competencias socioemocionales desde observadores externos o desde la percepción del profesor (Sweet, Guthrier y Mary, 1998). En general, no suelen partir de las percepciones de los alumnos.

En concreto, este trabajo se propone evaluar las características del profesor que resultan motivadoras para los alumnos de secundaria, a partir de su propia percepción. Se ha optado por la visión de los alumnos, con las limitaciones que puede tener, a cambio de la obtención de índices objetivos de conocimiento del profesor. El uso de autoinformes de los alumnos, como manifiesta Wentzel (2002), aporta un conocimiento como consecuencia de estar un año completo conviviendo con un profesor. Este tipo de familiaridad no es posible mediante otros métodos, que aportan evaluaciones en un momento puntual, valorando de diferente manera los aspectos socioemocionales presentes en el día a día del aula.

El presente estudio también se plantea valorar si existen diferencias en función de los diferentes cursos (edad), y del sexo; además, se pretende analizar posibles diferencias en las características motivantes señaladas por los alumnos y los factores de motivación académica que manifiestan los mismos. 


\section{Método}

La muestra, extraída de forma incidental, está compuesta por 350 alumnos de ESO matriculados en dos centros concertados, de diferentes áreas de la Comunidad de Madrid. Su distribución por curso, edad y sexo puede verse en la tabla 1.

TABLA 1. Distribución de alumnos por curso, edad y sexo: Frecuencias y porcentajes.

\begin{tabular}{|l|c|c|}
\hline CURSO & Frecuencia & $\%$ \\
\hline Primero & 85 & 24,3 \\
\hline Segundo & 82 & 23,4 \\
\hline Tercero & 97 & 27,7 \\
\hline Cuarto & 86 & 24,6 \\
\hline Total & 350 & 100,0 \\
\hline
\end{tabular}

\begin{tabular}{|c|c|r|}
\hline EDAD & Frecuencia & $\%$ \\
\hline 12 & 48 & 13,7 \\
\hline 13 & 80 & 22,9 \\
\hline 14 & 86 & 24,6 \\
\hline 15 & 79 & 22,6 \\
\hline 16 & 49 & 14,0 \\
\hline 17 & 7 & 2,0 \\
\hline 18 & 1 & 0,3 \\
\hline Total & 350 & 100,0 \\
\hline
\end{tabular}

\begin{tabular}{|l|c|c|}
\hline SEXO & Frecuencia & $\%$ \\
\hline Hombre & 167 & 47,7 \\
\hline Mujer & 183 & 52,3 \\
\hline Total & 350 & 100,0 \\
\hline
\end{tabular}

A los alumnos se les aplicó la escala de motivación académica (EMA, adaptada de Manassero y Vázquez, 1997, 1998) y la escala CPM, acerca de las características motivadoras de los profesores, elaborada ad-casum (Ver anexo 1).

La escala EMA es un inventario de 28 ítems que reflejan diferentes razones para justificar la asistencia a clase. La escala plantea una serie de respuestas a la pregunta "¿Por qué vas al colegio/instituto?". Los alumnos han de valorar el grado de correspondencia de cada una de las razones presentadas, en una escala tipo likert de 1 a 7 , reflejando su opinión personal. Su coeficiente de fiabilidad alcanza un valor Alfa de Cronbach de $0.88^{1}$.

Estudios anteriores (Núñez y Fontana, 2009) revelaron diferencias en la factorización de los elementos de la escala en comparación con los estudios de referencia (Manassero y cols., 1997, 1998). Se tomarán tales factores para presentar los hallazgos del presente trabajo. De esta manera, las variables definidas en el EMA, cuya caracterización se recoge en la tabla 2, serán: Motivación Extrínseca Internalizada, Motivación Extrínseca Pura, Desmotivación, Motivación Intrínseca Pura, Motivación Intrínseca Vocacional.

La escala CPM presenta un inventario de características del profesor entre las cuales los alumnos han de elegir las tres que más les motiven. También se les presentó una pregunta abierta en la que debían reflejar "otras características" que a su juicio resultan facilitadoras de la motivación.

Las escalas fueron aplicadas a los alumnos de forma colectiva y en una sola sesión.

En relación al análisis estadístico de los datos recogidos, se realizaron contrastes de "Chi-cuadrado" para valorar las diferencias entre las frecuencias obtenidas en función de los cursos y el sexo. Además, se muestran las aportaciones de los alumnos en relación a qué

1. Remitimos a trabajos anteriores (Manassero y cols., 1997, 1998; Núñez y Fontana, 2009) para conocer en profundidad las características técnicas. 
otras característica del profesor consideran motivantes. Finalmente, se presentan las frecuencias de elección de las características de los profesores en función de los factores de la escala EMA.

TABLA 2. Denominación y caracterización de los factores e ítems de la escala EMA.

\begin{tabular}{|l|l|l|l|}
\hline Factor & \multicolumn{1}{|c|}{ Denominación } & \multicolumn{1}{c|}{ Caracterización } & \multicolumn{1}{c|}{ Ítems } \\
\hline F1: MEI & $\begin{array}{l}\text { Motivación externa } \\
\text { internalizada }\end{array}$ & $\begin{array}{l}\text { Referencia a notas, demostración de } \\
\text { valía y de inteligencia }\end{array}$ & $\begin{array}{l}28,7,27,21,13, \\
14,6\end{array}$ \\
\hline F2: MEP & Motivación externa & $\begin{array}{l}\text { Referencia a prestigio laboral, ganar } \\
\text { más dinero, y superación de retos }\end{array}$ & $22,8,15,1,10$ \\
\hline F3: D & Desmotivación & No saber por qué se va al colegio & $19,26,5,12$ \\
\hline F4: MIP & Motivación Interna & Gusto de conocer & 18,11 \\
\hline F5: MIV & $\begin{array}{l}\text { Motivación } \\
\text { Intrínseca-Vocacional }\end{array}$ & $\begin{array}{l}\text { Recoge aspectos vocacionales, } \\
\text { referidos a intereses e inquietudes, } \\
\text { elegir la carrera y el trabajo } \\
\text { deseados }\end{array}$ & $\begin{array}{l}9,23,25,16,17,3,2 \\
4,24,2\end{array}$ \\
\hline
\end{tabular}

\section{Resultados}

Para realizar el análisis de los datos se utilizó por un lado el paquete SPSS/PC+ (versión 15.0), calculando los valores de "Chi-cuadrado" a través del Excell.

La tabla 3 muestra el porcentaje de selección de cada una de las características del profesor que los alumnos han valorado como más motivadoras, su posición y las frecuencias de ocurrencia para cada uno de los cursos y para cada sexo, así como los valores de "Chicuadrado" obtenidos.

La lectura atenta de los datos generales permite constatar gran coincidencia entre la percepción de los alumnos y los factores destacados en la literatura:

- la claridad de la exposición (ítem $6,1^{\circ}$ ), junto a la comprensión de los errores como parte del aprendizaje (ítem $9,10^{\circ}$ ), la exigencia en función de la capacidad (ítem 3 , $7^{\circ}$ ) y la información sobre los progresos (ítem $7,8^{\circ}$ ), tal y como señalan los estudios de Schweinle, Meyer y Turner (2006), en los que se destaca la necesidad de proveer de suficiente feedback y clarificación de conceptos y dudas;

- la valoración del esfuerzo y no sólo de los resultados (ítem $22,2^{\circ}$ ), la confianza en la capacidad de superar las dificultades (ítem 15, $3^{\circ}$ ), sentirse "importante" para el profesor (ítem $12,4^{\circ}$ ), podrían englobarse dentro de lo que se ha definido como una buena relación profesor-alumno (Skinner y Belmont, 1993; Connel y Wellborn, 1991, citado en Murray y Greenberg, 2000), destacando el papel del afecto, como factor esencial para la experiencia que viven los estudiantes en clase (Schweinle y cols., 2006);

- la conversión del aula en un lugar apasionante (ítem $4,5^{\circ}$ ), con actividades sorprendentes (ítem 16, $6^{\circ}$ ), que se traduce en la generación de curiosidad en el alumno, apoyaría los hallazgos de dos estudios realizados por Patrick, Hisley y Kempler 
(2000), en los que se exploran los efectos del entusiasmo del profesor, demostrando su relación con la motivación intrínseca por aprender y la vitalidad psicológica;

- promover un ambiente de cooperación (ítem $1,9^{\circ}$ ) entre los alumnos y e incitar la participación en el aula (ítem $18,11^{\circ}$ ) se han señalado como factores motivadores, favoreciendo la autonomía y las relaciones sociales (Schweinle y cols., 2006).

TABLA 3. Frecuencia (porcentaje), posición y valores de Chi cuadrado ( ${ }^{*} \alpha=0.05$; ${ }^{*} \alpha=0.01$ ) para cada ítem, en función del curso y del sexo.

\begin{tabular}{|c|c|c|c|c|c|c|c|c|c|c|}
\hline Ítem & $\%$ & posición & $\begin{array}{c}1^{\circ} \\
\text { ESO }\end{array}$ & $\begin{array}{c}2^{\circ} \\
\text { ESO }\end{array}$ & $\begin{array}{c}3^{\circ} \\
\text { ESO }\end{array}$ & $\begin{array}{c}4^{\circ} \\
\text { ESO }\end{array}$ & Chi & varón & mujer & Chi \\
\hline 1 & 16.72 & 9 & 16 & 19 & 15 & 7 & 6.29 & 28 & 29 & .046 \\
\hline 2 & 6.74 & & 5 & 4 & 8 & 6 & 0.85 & 4 & 19 & $8.47^{\text {** }}$ \\
\hline 3 & 18.18 & 7 & 15 & 15 & 19 & 14 & 0.32 & 38 & 24 & $4.59^{*}$ \\
\hline 4 & 24.63 & 5 & 18 & 19 & 22 & 25 & 1.29 & 40 & 44 & 0.00 \\
\hline 5 & 3.81 & & 4 & 7 & 1 & 1 & $8.76^{*}$ & 2 & 11 & $5.44^{*}$ \\
\hline 6 & 32.84 & 1 & 23 & 24 & 39 & 26 & 2.98 & 50 & 62 & 0.42 \\
\hline 7 & 17.01 & 8 & 19 & 14 & 14 & 11 & 2.74 & 26 & 32 & 0.19 \\
\hline 8 & 1.47 & & 1 & 2 & 1 & 1 & 0.78 & 4 & 1 & 2.09 \\
\hline 9 & 13.78 & 10 & 7 & 15 & 14 & 11 & 3.27 & 24 & 23 & 0.21 \\
\hline 10 & 7.04 & & 5 & 3 & 9 & 7 & 2.37 & 11 & 13 & 0.03 \\
\hline 11 & 3.52 & & 1 & 1 & 3 & 7 & $8.00^{*}$ & 9 & 3 & 3.58 \\
\hline 12 & 25.81 & 4 & 23 & 21 & 22 & 22 & 0.37 & 38 & 50 & 0.72 \\
\hline 13 & 5.28 & & 4 & 5 & 6 & 3 & 0.85 & 9 & 9 & 0.04 \\
\hline 14 & 7.62 & & 5 & 4 & 8 & 9 & 2.14 & 15 & 11 & 1.04 \\
\hline 15 & 27.27 & 3 & 24 & 26 & 28 & 15 & 3.82 & 35 & 58 & $3.78^{*}$ \\
\hline 16 & 22.87 & 6 & 21 & 10 & 17 & 30 & $11.03^{*}$ & 34 & 44 & 0.53 \\
\hline 17 & 8.50 & & 7 & 8 & 9 & 5 & 0.97 & 13 & 16 & 0.10 \\
\hline 18 & 10.26 & 11 & 9 & 7 & 5 & 14 & 5.85 & 25 & 10 & $7.90^{* *}$ \\
\hline 19 & 7.62 & & 10 & 8 & 5 & 3 & 5.23 & 17 & 9 & 3.26 \\
\hline 20 & 2.64 & & 1 & 1 & 2 & 5 & 4.83 & 4 & 5 & 0.04 \\
\hline 21 & 0.88 & & 2 & 1 & 0 & 0 & 3.91 & 1 & 2 & 0.25 \\
\hline 22 & 31.96 & 2 & 28 & 23 & 30 & 28 & 0.39 & 51 & 58 & 0.04 \\
\hline 23 & 2.64 & & 2 & 1 & 5 & 1 & 3.78 & 5 & 4 & 0.22 \\
\hline 24 & 0.59 & & 1 & 0 & 0 & 1 & 2.09 & 2 & 0 & 2.19 \\
\hline
\end{tabular}

En cuanto a las variables curso y sexo se han constatado diferencias significativas. En concreto, los alumnos de $2^{\circ}$ resaltan el ítem 5 ("me deja tiempo suficiente para contestar"), y los alumnos de $4^{\circ}$ destacan los ítems 16 y 11 (la curiosidad a través de la sorpresa y la promoción de la competitividad); respecto del sexo, las alumnas destacan la confianza en la superación de las dificultades (ítem 15), disponer del tiempo necesario para contestar una pregunta realizada (ítem 5) y la vinculación de la materia con la propia vida (ítem 2), mientras que los varones resaltan la exigencia en función de la capacidad (ítem 3) y la invitación a participar en clase (ítem 18). 
El análisis cualitativo de las respuestas de los alumnos a la pregunta abierta permitió la clasificación de las aportaciones en 5 categorías (ver tabla $4^{2}$ ): cualidades del profesor y relación personal; aspectos relacionados con el apoyo, refuerzo y reconocimiento académicos; conductas "no"; aspectos didácticos, y, por último, "miscelánea".

El primer comentario ante los datos recogidos se refiere al número de aportaciones. De 350 alumnos que respondieron al cuestionario, pocos aprovecharon la oportunidad de ampliar la información (muchos repitieron características ya incluidas o la dejaron en blanco). En sus respuestas, los alumnos insisten en la necesidad de vinculación con el profesor, solicitando una atención más personalizada como medio de incrementar su motivación. Reclaman la propuesta de actividades más dinámicas, alegres y divertidas, así como la transmisión de la "pasión" por la materia que imparte y la actividad que realiza (un tema relevante para la reflexión del docente).

Además señalan aspectos interesantes al considerar las actividades de apoyo: indican el uso de premios y/o concursos para animar la participación de los estudiantes, pero añaden la sencilla (y sincera) felicitación por el trabajo bien realizado, así como la importancia de animar ante las dificultades, sin resolvérselas.

La categoría "NO" merece un comentario especial y quizás sea necesaria una profunda reflexión acerca de cómo los alumnos se sienten tratados en algunas ocasiones. No se trata de una reivindicación numerosa, ni generalizada, pero no deberían haber surgido aportaciones del tipo: "no insultar", "no humillar", "no gritar".

Sugerentes resultan las respuestas englobadas en "aspectos didácticos". Además de factores como el juego, las prácticas/experimentos y el trabajo en equipo, los alumnos señalan, claramente, la necesidad de plantear actividades novedosas, actuales, e interesantes.

Finalmente, se exponen los resultados en relación a los factores de la escala EMA (ver tabla 5). Para facilitar su análisis las puntuaciones obtenidas en los factores se han categorizado en 3 niveles ${ }^{3}$. Las dimensiones de motivación que pueden caracterizar, en general, a esta muestra de alumnos son: factor de motivación extrínseca fuerte, tanto en la interiorizada (MEI), como en la "pura" (MEP); pobre factor de desmotivación (D); factor intrínseco puro entre medio y débil (siendo excepcionales los casos que manifiestan un factor alto de MIP); y, finalmente, un factor de motivación intrínseca-vocacional con tendencia a fuerte (MIV).

En general, no se han encontrado patrones de motivación diferenciales en relación a las características del profesor que los alumnos han señalado como facilitadoras de su motivación. Sin embargo, puede hablarse de una tendencia general en la que se pone de manifiesto la prevalencia de la motivación extrínseca (MEP). Después, surgen dos patrones. En el primero, se observa mayor frecuencia del factor de motivación vocacional (MIV), seguido en tercer lugar del factor de motivación externa internalizada (MEI). Como ejemplo pueden consultarse los datos de los ítems 4, 6 y 22. En el segundo, la frecuencia de ambos tipos de motivación (MIV y MEI) se iguala (ver ítems 12 y 15). La cuarta posición siempre corresponde a la motivación intrínseca pura (MIP), y la última a la desmotivación (D).

2. Aunque algunas de las características señaladas pueden parecer similares, se ha respetado el lenguaje utilizado por los alumnos para no reducir la información aportada.

3. Puntuación en el factor de 1 a 2,9: categoría 1, nivel bajo; de 3 a 5: categoría 2, nivel medio; de 5,1 a 7: categoría 3 , nivel alto. 
TABLA 4. Respuestas de los alumnos por cursos a la pregunta abierta: "Otras características que me parecen facilitadoras de la motivación son", categorizadas en cinco dimensiones: Cualidades y relación personal, Refuerzo/apoyo académico, Aspectos didácticos, Conductas “NO" y Miscelánea.

\begin{tabular}{|c|c|c|c|c|c|c|c|c|c|}
\hline \multirow{2}{*}{$\begin{array}{c}\text { CUALIDADES Y RELACIÓN } \\
\text { PERSONAL }\end{array}$} & \multicolumn{4}{|c|}{ Cursos } & \multirow{2}{*}{ REFUERZOIAPOYO ACADÉMICO } & \multicolumn{4}{|c|}{ Cursos } \\
\hline & $1^{\circ}$ & $2^{\circ}$ & $3^{\circ}$ & $4^{\circ}$ & & $1^{\circ}$ & $2^{\circ}$ & $3^{\circ}$ & $4^{\circ}$ \\
\hline Apoyo personal & 3 & 2 & 1 & 2 & Ayuda a mejorar (oportunidades) & 2 & 8 & 6 & 3 \\
\hline Amabilidad/Confianza & 6 & 2 & 3 & 1 & Felicitaciones & 1 & & 2 & 1 \\
\hline Respeto & 1 & & & 1 & Premios & 1 & 1 & & \\
\hline Paciencia & 1 & 1 & 2 & & Nunca se puede sacar más nota & 1 & & & \\
\hline Interés/comprensión & 3 & 3 & 4 & 3 & Exigencia & & 3 & 2 & \\
\hline Control del aula & 1 & & 1 & & Confianza en mi superación & & 3 & 2 & 1 \\
\hline Escucha & 2 & & & & Animar sin resolver & & 6 & 1 & 1 \\
\hline Personalidad & 2 & & & & Concurso & & & 1 & 1 \\
\hline Relación personal & 5 & 4 & 3 & 3 & Dificultad del examen (reto) & & & & 1 \\
\hline Bueno & & & 2 & & \multirow{2}{*}{ ASPECTOS DIDÁCTICOS } & \multicolumn{4}{|c|}{ Cursos } \\
\hline Afecto & & & 2 & & & $1^{\circ}$ & $2^{\circ}$ & $3^{\circ}$ & $4^{\circ}$ \\
\hline Majo & & & 1 & 1 & Trabajo en grupos & 1 & 1 & 1 & 1 \\
\hline Empatía & & & 1 & 1 & Dudas & 1 & 1 & 2 & 1 \\
\hline Pregunta lo enseñado & & & 1 & 2 & Ambiente (rollo) & 1 & & 2 & 1 \\
\hline Cariño & & & & 1 & Participación & 2 & 3 & 4 & 2 \\
\hline Neutralidad & & & & 1 & Pistas & 1 & & & \\
\hline Humor (buen) & 1 & & & 1 & Prácticas-experimentos & 3 & 1 & & \\
\hline Divertido & 6 & 3 & & & Juegos & & 1 & & \\
\hline Dinámico & 1 & & & & Esquemas-resúmenes & & 1 & 1 & \\
\hline Simpatía & & & 2 & & ejemplos & & & 2 & \\
\hline Nos / Él disfrute & & 2 & & & Atractivas/interesantes/novedosas/actuales & & 2 & 18 & 8 \\
\hline Agradable & & & & 1 & Valoración crítica & & & & 1 \\
\hline Amistosos & & & & 1 & Forma de dar clase & & & & 1 \\
\hline Alegre & & & & 1 & Debates/creativas/contenidos más abiertos & & & & 1 \\
\hline \multirow{2}{*}{ CONDUCTAS “NO” } & \multicolumn{4}{|c|}{ CURSOS } & \multirow{2}{*}{ CONDUCTAS “NO” } & \multicolumn{4}{|c|}{ CURSOS } \\
\hline & $1^{\circ}$ & $2^{\circ}$ & $3^{\circ}$ & $4^{\circ}$ & & $1^{0}$ & $2^{\circ}$ & $3^{\circ}$ & $4^{\circ}$ \\
\hline No perder paciencia & 1 & & & & No gritos & & 1 & 1 & \\
\hline No regañar & 2 & 1 & & & No insultos & & 2 & & \\
\hline No humillar en público & 1 & & & & No borde & & & 1 & 1 \\
\hline No enfadar & 1 & & & & No amargado & & & 2 & \\
\hline No estricto & 1 & & & & Mal humor & & & & 1 \\
\hline
\end{tabular}


TABLA 4. (Continuación).

\begin{tabular}{|l|c|c|c|c|}
\hline \multirow{2}{*}{ MISCELÁNEA } & \multicolumn{3}{c|}{ Cursos } \\
\cline { 2 - 5 } & $\mathbf{1}^{\circ}$ & $\mathbf{2}^{\mathbf{0}}$ & $\mathbf{3}^{\circ}$ & $\mathbf{4}^{\mathbf{0}}$ \\
\hline Explica muy bien & 6 & & & \\
\hline Sabe explicar & 3 & & & \\
\hline Deberes atractivos & 1 & & & \\
\hline Entiende y razona los errores & 3 & & & \\
\hline Temas interesantes, que llaman la atención & 3 & 1 & & \\
\hline Ejemplos de la vida cotidiana & 1 & & & \\
\hline Nunca se puede sacar más nota & 1 & & & \\
\hline Sentido y utilidad de los temas & & 1 & & \\
\hline Calificación (trabajos, no sólo exámenes) Nervios & & 1 & 2 & \\
\hline Excursiones & & 1 & & \\
\hline Música para relajar & & 2 & & \\
\hline Ejemplos de la vida cotidiana & & & 1 & 1 \\
\hline Trato más personal, clases menos numerosas & & & & 1 \\
\hline Gusto por la materia y por enseñarla; satisfacción por su labor & & & & 4 \\
\hline Programa claro y ordenado & & & & 1 \\
\hline Menos uso del libro de texto & & & & 3 \\
\hline Explicación que capte la atención & & & & 2 \\
\hline Competitividad & & & & 1 \\
\hline Invitación a investigar, preguntas e inquietudes & & & 2 \\
\hline
\end{tabular}

\section{Conclusiones}

Los resultados encontrados son consistentes con la literatura que subraya la importancia de la percepción de los alumnos en el conocimiento de qué características de un profesor favorecen su motivación por aprender (Murray y Greenberg, 2000). Se podrían destacar tres aspectos: el primero tiene que ver con el modo en que el profesor aborda el proceso de aprendizaje ofreciendo el feedback necesario y clarificando dudas y errores de forma que favorezca la implicación del alumno en su propio proceso (Schweinle y cols., 2006); el segundo hace referencia a la relación educativa entre profesor y alumno como factor decisivo de su motivación; por último, el tercer bloque de características permiten reconocer dos aspectos metodológicos fundamentales: despertar la curiosidad de los alumnos haciendo las clases apasionantes y fomentar la cooperación entre ellos como método de trabajo.

- Manifestar elevadas expectativas en relación a su capacidad y la devolución de un feed-back constructivo (Wentzel, 1997; 2002; Schweinle y col., 2006).

- Cuidar la dimensión de relación profesor-alumno, con especial atención a la expresión del afecto (Schweinle y col., 2006; Skinner y col. 1993; Connel y col, 1991). 
TABLA 5. Frecuencias de elección de características motivantes en función de los factores de la escala CPM categorizados: MEIc, MEc, DMc, MIPc, MIVc.

\begin{tabular}{|c|c|c|c|c|c|c|c|c|c|c|c|c|c|c|c|}
\hline IT & \multicolumn{3}{|c|}{ MEIC } & \multicolumn{3}{|c|}{ MEPc } & \multicolumn{3}{|c|}{ DMc } & \multicolumn{3}{|c|}{ MIPC } & \multicolumn{3}{|c|}{ MIVc } \\
\hline CPM & 1 & 2 & 3 & 1 & 2 & 3 & 1 & 2 & 3 & 1 & 2 & 3 & 1 & 2 & 3 \\
\hline 1 & 3 & 23 & 31 & 1 & 8 & 48 & 48 & 8 & 0 & 20 & 31 & 5 & 2 & 22 & 31 \\
\hline 2 & 1 & 14 & 8 & 3 & 5 & 13 & 20 & 2 & 0 & 6 & 14 & 2 & 0 & 8 & 13 \\
\hline 3 & 5 & 26 & 30 & 0 & 10 & 50 & 51 & 10 & 0 & 33 & 25 & 3 & 5 & 26 & 28 \\
\hline 4 & 5 & 38 & 36 & 2 & 19 & 62 & 72 & 11 & 0 & 28 & 51 & 5 & 4 & 27 & 50 \\
\hline 5 & 2 & 7 & 4 & 1 & 3 & 8 & 7 & 5 & 1 & 6 & 5 & 1 & 4 & 6 & 2 \\
\hline 6 & 7 & 47 & 55 & 1 & 29 & 82 & 96 & 14 & 1 & 47 & 60 & 5 & 4 & 41 & 63 \\
\hline 7 & 8 & 21 & 26 & 2 & 12 & 42 & 52 & 6 & 0 & 26 & 30 & 2 & 6 & 18 & 30 \\
\hline 8 & 1 & 0 & 4 & 0 & 1 & 4 & 3 & 1 & 1 & 2 & 3 & 0 & 1 & 0 & 4 \\
\hline 9 & 2 & 19 & 24 & 0 & 14 & 33 & 37 & 8 & 0 & 23 & 22 & 2 & 2 & 21 & 22 \\
\hline 10 & 1 & 7 & 14 & 1 & 4 & 19 & 21 & 3 & 0 & 10 & 13 & 1 & 1 & 7 & 14 \\
\hline 11 & 3 & 4 & 5 & 0 & 2 & 10 & 9 & 3 & 0 & 4 & 8 & 0 & 1 & 4 & 6 \\
\hline 12 & 5 & 38 & 44 & 0 & 22 & 62 & 75 & 9 & 1 & 39 & 43 & 5 & 2 & 31 & 47 \\
\hline 13 & 0 & 11 & 7 & 0 & 3 & 15 & 13 & 4 & 0 & 7 & 11 & 0 & 0 & 10 & 7 \\
\hline 14 & 3 & 11 & 12 & 1 & 4 & 21 & 22 & 4 & 0 & 10 & 16 & 0 & 2 & 10 & 14 \\
\hline 15 & 3 & 31 & 55 & 1 & 21 & 71 & 78 & 14 & 1 & 33 & 56 & 4 & 3 & 31 & 57 \\
\hline 16 & 11 & 34 & 29 & 3 & 16 & 56 & 68 & 7 & 1 & 23 & 49 & 5 & 4 & 33 & 38 \\
\hline 17 & 2 & 13 & 12 & 0 & 6 & 22 & 25 & 3 & 1 & 9 & 18 & 2 & 3 & 11 & 14 \\
\hline 18 & 2 & 19 & 14 & 0 & 5 & 30 & 28 & 6 & 0 & 16 & 19 & 0 & 0 & 18 & 16 \\
\hline 19 & 1 & 10 & 13 & 0 & 5 & 21 & 19 & 7 & 0 & 9 & 16 & 0 & 0 & 10 & 15 \\
\hline 20 & 2 & 5 & 2 & 1 & 4 & 4 & 6 & 2 & 0 & 2 & 7 & 0 & 0 & 5 & 4 \\
\hline 21 & 0 & 2 & 1 & 1 & 1 & 1 & 2 & 1 & 0 & 2 & 1 & 0 & 1 & 1 & 1 \\
\hline 22 & 11 & 47 & 47 & 3 & 29 & 75 & 91 & 14 & 1 & 42 & 60 & 5 & 2 & 47 & 56 \\
\hline 23 & 2 & 5 & 2 & 0 & 2 & 7 & 6 & 2 & 1 & 7 & 1 & 1 & 1 & 4 & 4 \\
\hline 24 & 1 & 0 & 1 & 0 & 0 & 2 & 2 & 0 & 0 & 1 & 1 & 0 & 0 & 2 & 0 \\
\hline
\end{tabular}

A partir de los hallazgos de este estudio, pueden señalarse ciertas pautas de actuación recomendables para todo profesor que pretenda mejorar los índices de motivación de sus alumnos, tales como las siguientes: 
- Transmitir pasión por la labor realizada, a través de la conversión del aula en un verdadero centro de aprendizaje dinámico y sorprendente (Patrick y col, 2000).

- Promover actividades que desarrollen el aprendizaje cooperativo y utilizar estrategias de control de clase positivas (Hawkins y cols. 1992).

En cuanto a las limitaciones del estudio, es evidente que las percepciones de los alumnos están mediadas por diversos aspectos entre los que cabe destacar y sería necesario estudiar: las propias características individuales de los alumnos, las del profesor y las de la escuela, factor este último menos considerado en su relación con la motivación.

Convendría, como línea futura de investigación, ampliar el estudio atendiendo las relaciones profesores-padres, para conocer qué peso tiene unas y otras. De hecho, algún trabajo valora la relevancia de los modelos de socialización parental para entender las relaciones entre las prácticas de los profesores y la adaptación escolar de los adolescentes. En concreto, Wentzel (2002) señala que es probable que los profesores tengan una mayor influencia en la motivación y conducta en el aula que los padres. Sin duda son necesarios estudios que examinen las contribuciones relativas de los contextos parentales, de enseñanza y de iguales, en la motivación de los estudiantes, estando especialmente indicada la perspectiva longitudinal.

Sería interesante llegar a establecer relaciones causales, imposibles con este estudio descriptivo, ya que resultarían de gran utilidad para favorecer la motivación de los alumnos, uno de los componentes claves de la competencia socio-emocional.

\section{Referencias Bibliográficas}

Bowlby, J. (1982). Attachment and loss: Volume I (2nd ed.). New York: Basic Books.

Brier, N. (2006). Enhancing academic motivation: an intervention program for young adolescents. Champaign, IL, US: Research Press.

Broc, M. A. (2006). Motivación y rendimiento académico en alumnos de Educación Secundaria Obligatoria y Bachillerato LOGSE. Revista de Educación, 340, 379-414.

Bueno, J. A. (2004). La motivación del alumno en el aula. Madrid: ICCE.

Ford, D. H. y Lerner, R. M. (1992). Development Systems Theory: An integrated approach. Newbury Park, CA: Sage.

Goleman, D. (1996). Inteligencia Emocional. Barcelona: Kairós.

González, R.; Mendiri, P. y Arias, A. (2002). Una aproximación a las variables predictoras del rendimiento académico en estudiantes de secundaria. Revista Española de Orientación y Psicopedagogía, 13 (2), 223-235.

Hawkins, J. D., Catalano, R. F. y Miller, J. Y. (1992). Risk and protective factors fro alcohol and other drug problems in adolescence and early adulthood: Implications for substance abuse prevention. Psychological Bulletin, 112, 64-105.

Hirsch, T. (1969). Causes of delinquency. Berkeley, CA: University of California Press.

Manassero, M. A. y Vázquez, A. (1997). Análisis empírico de dos escalas de motivación escolar. Revista Electrónica de Motivación y Emoción, 3 (5-6).

Manassero, M. A. y Vázquez, A. (1998). Validación de una escala de motivación de logro. Psicothe$m a, 10$ (2), 333-351. 
Menec, V. H. (1997). Volition and motivation: The effect of distracting learning conditions on students differing in action control and perceived control. Dissertation Abstracts International: Section B: The Sciences and Engineering, 57 (10-B), 6654.

Murray, Ch. y Greenberg, M. T. (2000). Children's relationships with teachers and bond with school. An investigation of patterns and correlates in middle childhood. Journal of School Psychology, 38 (5), 423-445.

Núñez, M. C. y Fontana, M. (2009). Atribuciones causales y características del profesor que favorecen la motivación en alumno con fracaso escolar: Implicaciones para la orientación educativa. Actas del I Congreso Internacional de Orientación Psicoeducativa. Madrid. En prensa.

Patrick, B. C.; Hisley, J. y Kempler, T. (2000). "What's everybody so excited about?": The effect of teacher enthusiasm on students intrinsic motivation and vitality. Journal of Experimental Education, 68 (3), 217-236.

Patrick, H.; Mantzicopoulos, P.; Samarapungavan, A. y French, B. F. (2008). Patterns of young children's motivation of science and teacher-child relationships. Journal of Experimental Education, 76 (2), 121-144.

Pianta, R. C. (1999). Enhancing relationships between children and teachers. Washington, DC: American Psychological Association.

Resnick, M. D.; Bearman, P. S.; Blum, R. M.; Bauman, K. E.; Harris, K. M.; Jones, J.; Tabor, J.; Beuhring, T.; Sieving, R. E.; Shew, M.; Ireland, M.; Bearinger, L. H. y Udry, J. R. (1997). Protecting adolescents from harm: Findings from the National Longitudinal Study on Adolescent Health. The Journal of the American Medical Association, 278, 823-833.

Schweinle, A.; Meyer, D. K. y Turner, J. C. (2006). Striking the right balance: Students motivation and affect in elementary mathematics. Journal of Educational Research, 99 (5), 271-295.

Schiltz, L. (2004). Achievement motivation, academic destiny and coping strategies. Some implications of a comparative follow-up study. Neuropsychiatrie de l'Enfance et de Adolescence, 52 (2), 70-77.

Sekiro, Y. y Fantuzzo, J. (2005). Validity of the Child Observation Record: An investigation of the relationships between COR dimensions and social emotional and cognitive outcomes for Head Start Children. Journal of Psychoeducational Assessment, 23 (3), 242-260.

Skinner, E. A. y Belmont, M. J. (1993). Motivation in the classroom: reciprocal effects of teacher behaviour and student engagement across the year. Journal of Educational Psychology, 85, 571581.

Sweet, A. P.; Guthrie, J. T. y Mary, M. (1998). Teacher perceptions and student reading motivation. Journal of Educational Psychology, 90 (2), 210-223.

Wentzel, K. R. (1997). Student motivation in middle school. The role of perceived pedagogical caring. Journal of Educational Psychology, 89 (3), 411-419.

Wentzel, K. R. (1998). Social relationships and motivation in middle school: The role of parents, teachers and peers. Journal of Educational Psychology, 90 (2), 202-209.

Wentzel, K. R. (2002). Are effective teachers like good parents? Teaching styles and student adjustment in early adolescence. Child Development, 73 (1), 287-301.

Wooley, M. E., Kol, K. L. y Bowen, G. L. (2009). The social context of school success for latino Middle School students direct and indirect influences of teachers, family and friends. Journal of Early Adolescence, 29 (1), 43-70.

Fecha de recepción: 14-07-2009

Fecha de revisión: 21-09-2009

Fecha de aceptación: 21-10-2009 


\section{ANEXO 1: \\ Cuestionario acerca de las características del profesor motivante (CPM). Frecuencia de elección y posición relativa}

\begin{tabular}{|c|c|c|}
\hline & Posición & $\%$ \\
\hline 1. Crea en clase un ambiente de cooperación & 9 & 16.72 \\
\hline 2. Explica la relación que tiene conmigo todo lo que enseña & & 6.74 \\
\hline 3. Me exige en función de mi capacidad & 7 & 18.18 \\
\hline 4. Hace las clases apasionantes & 5 & 24.63 \\
\hline $\begin{array}{l}\text { 5. Cuando pregunta en clase, me deja tiempo suficiente para contestar la } \\
\text { pregunta }\end{array}$ & & 3.81 \\
\hline 6. Explica de manera clara, tratando de que comprenda los temas & 1 & 32.84 \\
\hline 7. Me informa de mis avances y progresos & 8 & 17.01 \\
\hline 8. Me critica cuando fallo en alguna pregunta o suspendo un examen & & 1.47 \\
\hline 9. Entiende mis errores como parte del aprendizaje & 10 & 13.78 \\
\hline $\begin{array}{l}\text { 10. Corrige las tareas que manda, señalando los progresos y necesidades } \\
\text { de mejora }\end{array}$ & & 7.04 \\
\hline 11. Promueve la competitividad en clase & & 3.52 \\
\hline $\begin{array}{l}\text { 12. Siento que le importo y que le importan mis inquietudes y problemas } \\
\text { para estudiar }\end{array}$ & 4 & 25.81 \\
\hline 13. Me da pistas cuando no sé la respuesta a una pregunta & & 5.28 \\
\hline 14. Expresa la relación que tiene un tema con los anteriores & & 7.62 \\
\hline 15. Confía en que soy capaz de superar las dificultades & 3 & 27.27 \\
\hline 16. Sus clases resultan sorprendentes, despiertan mi curiosidad & 6 & 22.87 \\
\hline 17. Utiliza medios audiovisuales como power point, transparencias, etc. & & 8.50 \\
\hline 18. Invita a participar en sus clases & 11 & 10.26 \\
\hline 19. Favorece la colaboración y la ayuda entre los alumnos & & 7.62 \\
\hline $\begin{array}{l}\text { 20. Su estilo se define por provocar muchas preguntas más que por dar } \\
\text { respuestas }\end{array}$ & & 2.64 \\
\hline 21. Si tardo en contestar una pregunta, rápidamente me da la respuesta & & 0.88 \\
\hline 22. Tiene en cuenta el esfuerzo que realizo y no sólo el resultado & 2 & 31.96 \\
\hline 23. En sus clases utilizamos, sobre todo, el libro de texto & & 2.64 \\
\hline 24. Manda muchas tareas que no nos devuelve corregidas & & 0.59 \\
\hline
\end{tabular}

